OU-HET 256

hep-th/9701095

\title{
NON-EXTREME BLACK HOLES FROM INTERSECTING M-BRANES
}

\author{
Nobuyoshi Ohta円 and Takashi Shimizu民̈ \\ Department of Physics, Osaka University, \\ Toyonaka, Osaka 560, Japan
}

\begin{abstract}
We investigate the possibility of extending non-extreme black hole solutions made of intersecting M-branes to those with two non-extreme deformation parameters, similar to Reissner-Nordstrøm solutions. General analysis of possible solutions is carried out to reduce the problem of solving field equations to a simple algebraic one for static spherically-symmetric case in $D$ dimensions. The results are used to show that the extension to two-parameter solutions is possible for $D=4,5$ dimensions but not for higher dimensions, and that the area of horizon always vanishes in the extreme limit for black hole solutions for $D \geq 6$ except for two very special cases which are identified. Various solutions are also summarized.
\end{abstract}

\footnotetext{
${ }^{1}$ e-mail address: ohta@phys.wani.osaka-u.ac.jp

${ }^{2}$ e-mail address: simtak@phys.wani.osaka-u.ac.jp
} 


\section{Introduction}

There has been much interest in solitonic and black hole solutions in string theories because of the possible resolution of various puzzles associated with quantum gravity [1, 2, 3, 4, 5, 6, 7]. The study of this subject has got its upsurge due to the recent discovery that the statistical origin of the entropy of BPS-saturated black holes can be addressed from the string point of view $[8,9,10,11,12,13,14,15,16]$.

By now many extreme as well as non-extreme solutions have been understood from the low-dimensional effective theory. There is now a consensus that the best candidate for a unified theory underlying all physical phenomena is no longer ten-dimensional string theory but rather eleven-dimensional M-theory. Though the precise formulation of M-theory is not known, its low energy limit coincides with the eleven-dimensional supergravity. Thus it is expected that the black hole solutions get their natural framework in the eleven-dimensional supergravity.

Indeed, the most transparent and systematic approach to this problem is provided by identifying these black holes as compactified configurations of intersecting two-branes and five-branes of eleven-dimensional supergravity [17]. It has been known that this theory admits various $p$-brane solutions [3], collectively referred to as M-branes. These solutions have been shown to be understood as the intersections of the fundamental twoand five-brane solutions [17, 18, 19, 20, 21, 22, 23, 24, 25.

Now rather systematic (the so-called "harmonic function") rule is developed to produce various extreme solutions as intersecting M-branes in eleven dimensions [18, and most of the known solutions can be derived systematically upon dimensional reduction to lower dimensions. This rule has also been extended to the one for non-extreme solutions with a single "non-extremality" parameter specifying a deviation from the BPS-limit [23]. It would be quite interesting to further elaborate on this approach and investigate how large a class of solutions are allowed.

In this paper we examine the possibility of extending non-extreme black hole solutions composed of intersecting M-branes in eleven dimensions to those with two deformation parameters, similar to the Reissner-Nordstrøm one in four dimensions. We perform a general analysis of possible solutions for the static spherically-symmetric case in $D$ di- 
mensions and summarize various typical solutions as well as new ones. Upon dimensional reduction, these eleven-dimensional solutions give rise to various non-extreme solutions in lower dimensions. In the extreme limit, most of these solutions give supersymmetric ones [26. I However, our analysis is not restricted to supersymmetric case and should be useful to searching for new solutions.

There has been a claim that there are no extreme black holes with regular horizons of finite area in $D \geq 6$ [6, 4, 21, 23]. Though no counter example to this claim has been discovered, general proof allowing the possibility of two non-extremality parameters has not been given even for the static case of our interest. This is also one of our motivations for trying to find solutions with two deformation parameters, since then we can keep a deformation parameter finite to obtain a horizon of finite area in the extreme limit. We use the results of the general analysis of the solutions to prove that the above claim is indeed true except only two solutions in $D=6,7$ dimensions. However, it can be shown that supersymmetry is not recovered in the "extreme" limit in the latter special solutions. If we require that there should be exact supersymmetry in the limit, our results indicate that there are no black holes with horizons of finite area in higher dimensions. We also discuss the universal expressions for the ADM mass and entropy of these black hole solutions.

The paper is organized as follows. In sect. 2, we summarize elements of elevendimensional supergravity relevant to our study, and collect various formulae used in the following sections. We then present our non-extreme black hole solutions in the form with two deformation parameters as intersecting M-branes for $D=4$ in sect. 3 and for $D=5$ in sect. 4 . The general analysis of possible solutions for $D \geq 6$ dimensions is presented in sect. 5 , where we also show that there are no solutions with two deformation parameters and that almost no extreme black holes can have regular horizons of finite area. In sect. 6, we summarize typical higher-dimensional solutions, including the sixand seven-dimensional solutions with regular horizons of finite area in the extreme limit. In sect. 7, we discuss the universal expressions for ADM mass and entropy. Sect. 8 is devoted to conclusions.

\footnotetext{
${ }^{1}$ It turns out that these solutions in $D=4,5$ dimensions are equivalent to known solutions $[3,23$ by redefinitions of variables and parameters to be discussed shortly.
} 


\section{Intersecting M-Branes}

In this section, we start with various definitions and conventions used in this paper. Our basic strategy is to look for the solutions in the low-energy limit of the M-theory, eleven-dimensional supergravity.

The bosonic parts of the field equations for eleven-dimensional supergravity are

$$
\begin{aligned}
R_{M N} & =\frac{1}{3}\left(F^{2}\right)_{M N}-\frac{1}{36} F^{2} g_{M N}, \\
\nabla_{M} F^{M N P Q} & =-\frac{1}{576} \epsilon^{N P Q M_{1} \cdots M_{8}} F_{M_{1} \cdots M_{4}} F_{M_{5} \cdots M_{8}}, \\
\partial_{[M} F_{N P Q R]} & =0 .
\end{aligned}
$$

where $M, N, \cdots=0,1, \cdots, 10$ are the curved-space indices and $\nabla_{M}$ is the covariant derivative. The last eq. in (2.1) is the Bianchi identity. It is also useful to note that $\nabla_{M} F^{M N P Q}=\partial_{M}\left(\sqrt{-g} F^{M N P Q}\right) / \sqrt{-g}$ where $g$ is the determinant of the metric.

The vanishing condition of the supersymmetry transformation of the gravitino gives the criterion for the existence of the unbroken supersymmetry. This is called the Killing equation for the Killing spinor $\zeta$ :

$$
\left[\partial_{M}+\frac{1}{4} \omega_{M}^{a b} \Gamma_{a b}+\frac{1}{144}\left(\Gamma_{M}^{N P Q R}-8 \delta_{M}^{N} \Gamma^{P Q R}\right) F_{N P Q R}\right] \zeta=0
$$

where $\omega_{M}^{a b}$ is the spin connection, $a, b$ are the tangent-space indices and $\Gamma$ 's are the antisymmetrized products of eleven-dimensional gamma matrices with unit strength. We are going to look for solutions for the field equations (2.1), and examine how supersymmetry remains unbroken by using the Killing equation (2.2).

We take the following metric for our system:

$$
d s_{11}^{2}=-e^{2 u_{0}} d t^{2}+e^{2 u_{1}}{\widehat{d y_{1}}}^{2}+\sum_{\alpha=2}^{d-1} e^{2 u_{\alpha}} d y_{\alpha}^{2}+e^{2 v} d r^{2}+e^{2 B} r^{2} d \Omega_{\tilde{d}+1}^{2},
$$

where the coordinate $\left(t, y_{\alpha}\right),(\alpha=1, \ldots, d-1)$ parametrize the $d$-dimensional worldvolumes of the intersecting M-branes with

$$
\widehat{d y_{1}}=d y_{1}+e^{w} d t
$$

The remaining coordinates of the eleven-dimensional spacetime are the radius $r$ and the angular coordinates on a $(\tilde{d}+1)=(10-d)$-dimensional unit sphere, whose metric is 
$d \Omega_{\tilde{d}+1}^{2}$. The off-diagonal component of the metric (2.4) is introduced in order to allow for the possibility of incorporating momentum along the M-brane space direction $\left(y_{1}\right)$. All the functions appearing in the metrics are assumed to depend only on the radius $r$ of the transverse dimensions, which, together with the time $t$, will be our eventual spacetime of dimension $D=\tilde{d}+3$.

Typically these metrics are chosen to be the products of various harmonic functions. The rule for identifying which coordinates belong to which brane is as follows [18]. If one divides out from the eleven-dimensional metric the overall conformal factor which multiplies the transverse spacetime, the coordinates multiplied with the same harmonic function belong to a given $p$-brane. We will soon see how this rule works.

In order to solve for our eqs. (2.1), we need the Ricci tensor for our metric (2.3). It is straightforward to derive the following results:

$$
\begin{aligned}
& R_{00}=e^{2\left(u_{0}-v\right)}\left[u_{0}^{\prime \prime}+u_{0}^{\prime}\left\{u_{0}^{\prime}+\sum_{\alpha=1}^{d-1} u_{\alpha}^{\prime}-v^{\prime}+(\tilde{d}+1)\left(B^{\prime}+\frac{1}{r}\right)\right\}\right] \\
& -e^{2\left(u_{1}+w-v\right)}\left[u_{1}^{\prime \prime}+w^{\prime \prime}+u_{0}^{\prime}\left(u_{1}^{\prime}-w^{\prime}\right)+2 u_{1}^{\prime} w^{\prime}+\frac{3}{2}\left(w^{\prime}\right)^{2}\right. \\
& \left.+\left(u_{1}^{\prime}+w^{\prime}\right)\left\{\sum_{\alpha=1}^{d-1} u_{\alpha}^{\prime}-v^{\prime}+(\tilde{d}+1)\left(B^{\prime}+\frac{1}{r}\right)\right\}\right] \\
& -\frac{\left(w^{\prime}\right)^{2}}{2} e^{2\left(-u_{0}+2 u_{1}-v+2 w\right)} \\
& R_{01}=-e^{2\left(u_{1}-v\right)+w}\left[u_{1}^{\prime \prime}+\frac{w^{\prime \prime}}{2}+u_{0}^{\prime}\left(u_{1}^{\prime}-\frac{w^{\prime}}{2}\right)\right. \\
& \left.+\left(u_{1}^{\prime}+\frac{w^{\prime}}{2}\right)\left\{\sum_{\alpha=1}^{d-1} u_{\alpha}^{\prime}+w^{\prime}-v^{\prime}+(\tilde{d}+1)\left(B^{\prime}+\frac{1}{r}\right)\right\}\right] \\
& -\frac{\left(w^{\prime}\right)^{2}}{2} e^{2\left(-u_{0}+2 u_{1}-v\right)+3 w} \\
& R_{11}=-e^{2\left(u_{1}-v\right)}\left[u_{1}^{\prime \prime}+u_{1}^{\prime}\left\{u_{0}^{\prime}+\sum_{\alpha=1}^{d-1} u_{\alpha}^{\prime}-v^{\prime}+(\tilde{d}+1)\left(B^{\prime}+\frac{1}{r}\right)\right\}\right] \\
& -\frac{\left(w^{\prime}\right)^{2}}{2} e^{2\left(-u_{0}+2 u_{1}-v+w\right)}, \\
& R_{\alpha \beta}=-e^{2\left(u_{\alpha}-v\right)}\left[u_{\alpha}^{\prime \prime}+u_{\alpha}^{\prime}\left\{u_{0}^{\prime}+\sum_{\gamma=1}^{d-1} u_{\gamma}^{\prime}-v^{\prime}+(\tilde{d}+1)\left(B^{\prime}+\frac{1}{r}\right)\right\}\right] \delta_{\alpha \beta}, \quad(\alpha, \beta>1) \\
& R_{r r}=-u_{0}^{\prime \prime}-u_{0}^{\prime}\left(u_{0}^{\prime}-v^{\prime}\right)-\sum_{\alpha=1}^{d-1}\left[u_{\alpha}^{\prime \prime}+u_{\alpha}^{\prime}\left(u_{\alpha}^{\prime}-v^{\prime}\right)\right]
\end{aligned}
$$




$$
\begin{gathered}
-(\tilde{d}+1)\left[B^{\prime \prime}+\frac{2 B^{\prime}-v^{\prime}}{r}-v^{\prime} B^{\prime}+\left(B^{\prime}\right)^{2}\right]+\frac{\left(w^{\prime}\right)^{2}}{2} e^{2\left(-u_{0}+u_{1}+w\right)} \\
R_{a b}=-e^{2(B-v)}\left[B^{\prime \prime}+\left(B^{\prime}+\frac{1}{r}\right)\left\{u_{0}^{\prime}+\sum_{\alpha=1}^{d-1} u_{\alpha}^{\prime}-v^{\prime}+(\tilde{d}+1)\left(B^{\prime}+\frac{1}{r}\right)\right\}-\frac{1}{r^{2}}\right] g_{a b} \\
+\frac{\tilde{d}}{r^{2}} g_{a b},
\end{gathered}
$$

where a prime denotes a derivative with respect to $r$, and $g_{a b}$ is the metric for $(\tilde{d}+1)$ sphere of radius $r$.

These formulae are valid not only for eleven-dimensional case but also for other dimensions. In particular, these will be useful for examining solutions for string theories in ten dimensions as well as the theories compactified in lower dimensions.

\section{$3 \quad D=4$ non-extreme solutions}

We first present our non-extreme solutions with two deformation parameters which correspond to $D=4$ black holes. In this section $\tilde{d}=1$. Four-dimensional extreme black holes with four charges can be derived from the two different intersecting M-brane configurations toroidally compactified [18, 22]: two two-branes and two five-branes $(2 \perp 2 \perp 5 \perp 5)$ and three five-branes with a boost along the common string $(5 \perp 5 \perp 5)$. Although our non-extreme solutions prove to be equivalent to known ones [3, 23], it is instructive to see the explicit forms and their relations. This also serves to establish our notation. We will be very brief.

\subsection{Non-extreme intersecting $2 \perp 2 \perp 5 \perp 5$ M-branes}

The first of the above eleven-dimensional configurations corresponds to the intersecting $2 \perp 2 \perp 5 \perp 5$. Our metric is given by

$$
\begin{aligned}
d s_{11}^{2}= & \left(H_{1} H_{2}\right)^{1 / 3}\left(H_{3} H_{4}\right)^{2 / 3}\left[-\left(H_{1} H_{2} H_{3} H_{4}\right)^{-1} f d t^{2}+H_{1}^{-1}\left(H_{3}^{-1} d y_{1}^{2}+H_{4}^{-1} d y_{2}^{2}\right)\right. \\
& +H_{2}^{-1}\left(H_{3}^{-1} d y_{3}^{2}+H_{4}^{-1} d y_{4}^{2}\right)+\left(H_{3} H_{4}\right)^{-1}\left(d y_{5}^{2}+d y_{6}^{2}+d y_{7}^{2}\right) \\
& \left.+f^{-1} d r^{2}+r^{2} d \Omega_{2}^{2}\right],
\end{aligned}
$$

which is a non-extreme generalization of the extreme solutions discussed in ref. [22] and the same form taken in ref. [23]. 
Our non-extreme deformation is parametrized by the two-parameter function

$$
f=\left(1-\frac{\mu_{1}}{r^{\tilde{d}}}\right)\left(1-\frac{\mu_{2}}{r^{\tilde{d}}}\right)
$$

The coordinates $y_{1}, \ldots, y_{7}$ will describe the toroidally compactified directions.

As explained in sect. 2, up to an overall factor, (the inverse of) the harmonic function $H_{1}$ multiplies the squares of the differentials of the coordinates $t, y_{1}$ and $y_{2}$, which means that these coordinates belong to a two-brane. Examining how other harmonic functions multiply the coordinates, one can easily see that this metric corresponds to a configuration of non-extreme intersecting $2 \perp 2 \perp 5 \perp 5$ M-branes.

We assume the following forms for our metric (3.1): :

$$
\begin{aligned}
F_{012 r} & =\frac{1}{2} T_{1}^{\prime} ; \quad F_{034 r}=\frac{1}{2} T_{2}^{\prime} ; \quad F_{24 a b}=\frac{1}{2} \epsilon_{a b r} T_{3}{ }^{\prime} ; \quad F_{13 a b}=-\frac{1}{2} \epsilon_{a b r} T_{4}{ }^{\prime} \\
H_{i} & =1+\frac{Q_{i}}{r}, \quad(i=1,2,3,4)
\end{aligned}
$$

where the indices $a$ and $b$ to the field strengths denote the angular coordinates of the transverse dimensions.

We substitute these into the field eqs. (2.1) and look for the solution for the field strength. We find the solution

$$
\begin{aligned}
& T_{i}=1-\frac{\sqrt{\left(Q_{i}+\mu_{1}\right)\left(Q_{i}+\mu_{2}\right)}}{r+Q_{i}}, \text { for } i=1,2, \\
& T_{j}=1-\frac{\sqrt{\left(Q_{j}+\mu_{1}\right)\left(Q_{j}+\mu_{2}\right)}}{r}, \text { for } j=3,4 .
\end{aligned}
$$

The non-extreme solutions discussed in ref. [23] correspond to $\mu_{2}=0$. Alternatively, if we make the redefinition

$$
\hat{r}^{\tilde{d}} \equiv r^{\tilde{d}}-\mu_{2} ; \quad \hat{Q}_{i} \equiv Q_{i}+\mu_{2} ; \quad \hat{\mu} \equiv \mu_{1}-\mu_{2}
$$

with $\tilde{d}=1$, our solution reduces to that in ref. 23]. In that case, the outer and inner horizons sit at $\hat{r}=\hat{\mu}^{1 / \tilde{d}}$ and 0 , and the extreme limit is given by $\hat{\mu} \rightarrow 0$. Thus the

\footnotetext{
${ }^{2}$ Throughout this paper, we use a prime to denote a derivative with respect to $r$, and never use it to distinguish different functions.

3 This was pointed out to us by R. Emparan and by I. Klebanov and A. Tseytlin.
} 
deformation parameter $\mu_{2}$ plays the role of shifting the horizon from $r=0$ to $\mu_{2}^{1 / \tilde{d}}$. An advantage of our expression is that it makes manifest that the area of the outer horizon is finite in the extreme limit.

Suppose $\mu_{1}>\mu_{2}$. It is convenient to parametrize

$$
Q_{i}=-\frac{\mu_{1}+\mu_{2}}{2}+\frac{\mu_{1}-\mu_{2}}{2} \cosh 2 \delta_{i}
$$

The nine-area at the outer horizon $r=\mu_{1}$ is then given by

$$
\begin{aligned}
A_{9} & =4 \pi L^{7}\left[r^{2}\left(H_{1} H_{2} H_{3} H_{4}\right)^{1 / 2}\right]_{r=\mu_{1}}=4 \pi L^{7} \prod_{i=1}^{4} \sqrt{Q_{i}+\mu_{1}} \\
& =4 \pi L^{7}\left(\mu_{1}-\mu_{2}\right)^{2} \prod_{i=1}^{4} \cosh \delta_{i},
\end{aligned}
$$

where $L$ is the (common) length of the coordinates $y_{\alpha}$.

The extreme limit is obtained by sending $\mu_{1}, \mu_{2} \rightarrow \mu$ while keeping $\left(\mu_{1}-\mu_{2}\right)^{1 / 2} \cosh \delta_{i}$ (or $Q_{i}$ ) finite. In what sense is this an "extreme limit"? Of course, the outer and inner horizons coincide in this limit. Moreover, examining the condition for the existence of non-zero Killing spinors (2.2), one finds that indeed 1/8 supersymmetry is preserved in the limit even for non-zero $\mu$. This is the same number of remaining supersymmetry for the extreme solution with $f=1$. In this limit, the nine-area becomes

$$
\left(A_{9}\right)_{B P S}=4 \pi L^{7} \sqrt{\left(Q_{1}+\mu\right)\left(Q_{2}+\mu\right)\left(Q_{3}+\mu\right)\left(Q_{4}+\mu\right)} .
$$

If we compactify $y_{1}, \cdots, y_{7}$, our solution reduces to the four-dimensional black hole with the Einstein-frame metric

$$
\begin{gathered}
d s_{4}^{2}=-\lambda(r) f(r) d t^{2}+\lambda^{-1}(r)\left[f^{-1}(r) d r^{2}+r^{2} d \Omega_{2}^{2}\right], \\
\lambda(r)=\left(H_{1} H_{2} H_{3} H_{4}\right)^{-1 / 2}=\frac{r^{2}}{\left[\left(r+Q_{1}\right)\left(r+Q_{2}\right)\left(r+Q_{3}\right)\left(r+Q_{4}\right)\right]^{1 / 2}} .
\end{gathered}
$$

Under the replacement (3.5), the four-dimensional metric (3.9) reduces to the solutions with two electric and two magnetic charges found in ref. [6, 23].

Dimensional reduction to $D=10$ along one direction common to the two five-branes, say $y_{7}$, gives type IIA solution representing R-R $p$-brane configuration $2 \perp 2 \perp 4 \perp 4$. T-duality along one of the two directions common to four-branes transforms this to the 
$3 \perp 3 \perp 3 \perp 3$ solution of type IIB. Reduction along $y_{1}$, on the other hand, produces the $1 \perp 2 \perp 4 \perp 5$ solution. Other solutions are similarly obtained by using T- and $S L(2, Z)$ duality.

\subsection{Non-extreme intersecting $5 \perp 5 \perp 5$ M-branes with boost}

The non-extreme generalization of the extreme $5 \perp 5 \perp 5$ M-branes with boost is given by

$$
\begin{aligned}
d s_{11}^{2}= & \left(H_{1} H_{2} H_{3}\right)^{2 / 3}\left[\left(H_{1} H_{2} H_{3}\right)^{-1}\left(-K^{-1} f d t^{2}+K{\widehat{d y_{1}}}^{2}\right)+\left(H_{2} H_{3}\right)^{-1}\left(d y_{2}^{2}+d y_{3}^{2}\right)\right. \\
& \left.+\left(H_{1} H_{3}\right)^{-1}\left(d y_{4}^{2}+d y_{5}^{2}\right)+\left(H_{1} H_{2}\right)^{-1}\left(d y_{6}^{2}+d y_{7}^{2}\right)+f^{-1} d r^{2}+r^{2} d \Omega_{2}^{2}\right]
\end{aligned}
$$

where

$$
\begin{aligned}
\widehat{d y_{1}} & =d y_{1}+\frac{\tilde{K}(r)}{K(r)} d t, \quad F_{23 a b}=\frac{1}{2} \epsilon_{a b r} T_{1}{ }^{\prime} ; \quad F_{45 a b}=\frac{1}{2} \epsilon_{a b r} T_{2}{ }^{\prime} ; \quad F_{67 a b}=\frac{1}{2} \epsilon_{a b r} T_{3}{ }^{\prime} \\
K(r) & =1+\frac{P}{r} ; \quad H_{i}(r)=1+\frac{Q_{i}}{r}, \quad(i=1,2,3) .
\end{aligned}
$$

Here $a$ and $b$ are again the coordinates of the transverse dimensions other than $r$, and $f$ is the same as eq. (3.2) with $\tilde{d}=1$, and the charge $P$ stands for the momentum along the common string in $y_{1}$ direction.

The solution to the field eqs. (2.1) is given by

$$
\tilde{K}=-\frac{\sqrt{\left(P+\mu_{1}\right)\left(P+\mu_{2}\right)}}{r} ; \quad T_{i}=1-\frac{\sqrt{\left(Q_{i}+\mu_{1}\right)\left(Q_{i}+\mu_{2}\right)}}{r}, \quad(i=1,2,3) .
$$

Like (3.6), we parametrize the charges by

$$
Q_{i}=-\frac{\mu_{1}+\mu_{2}}{2}+\frac{\mu_{1}-\mu_{2}}{2} \cosh 2 \delta_{i} ; \quad P=-\frac{\mu_{1}+\mu_{2}}{2}+\frac{\mu_{1}-\mu_{2}}{2} \cosh 2 \gamma .
$$

We again find the nine-area at the outer horizon $r=\mu_{1}$ to be

$$
\begin{aligned}
A_{9} & =4 \pi L^{7}\left[r^{2}\left(K H_{1} H_{2} H_{3}\right)^{1 / 2}\right]_{r=\mu_{1}}=4 \pi L^{7} \sqrt{\left(Q_{1}+\mu_{1}\right)\left(Q_{2}+\mu_{1}\right)\left(Q_{3}+\mu_{1}\right)\left(P+\mu_{1}\right)} \\
& =4 \pi L^{7}\left(\mu_{1}-\mu_{2}\right)^{2} \cosh \delta_{1} \cosh \delta_{2} \cosh \delta_{3} \cosh \gamma
\end{aligned}
$$

In the extreme limit $\mu_{1}, \mu_{2} \rightarrow \mu$ keeping $\left(\mu_{1}-\mu_{2}\right)^{1 / 2} \cosh \delta_{i}(\gamma)$ or $Q_{i}$ and $P$ finite, the nine-area becomes

$$
\left(A_{9}\right)_{B P S}=4 \pi L^{7} \sqrt{\left(Q_{1}+\mu\right)\left(Q_{2}+\mu\right)\left(Q_{3}+\mu\right)(P+\mu)} .
$$


The corresponding four-dimensional Einstein-frame metric is (3.9) with

$$
\lambda(r)=\left(H_{1} H_{2} H_{3} K\right)^{-1 / 2}=\frac{r^{2}}{\left[\left(r+Q_{1}\right)\left(r+Q_{2}\right)\left(r+Q_{3}\right)(r+P)\right]^{1 / 2}},
$$

in agreement with the metric found in ref. [6, 23] (under the replacement (3.5)) with one electric and three magnetic charges.

Compactification to $D=10$ along the direction $y_{1}$ common to three five-branes gives rise to type IIA solutions corresponding to three four-branes intersecting over twobranes plus additional Kaluza-Klein electric charge background, which may be called $0 \perp 4 \perp 4 \perp 4$ solution. T-duality transforms this solution to $2 \perp 2 \perp 4 \perp 4$ which is the dimensional reduction of the $2 \perp 2 \perp 5 \perp 5$ solution mentioned in the previous subsection. Upon compactification in other direction, say $y_{2}$, gives type IIA $5 \perp 4 \perp 4$ solution with boost. Again other solutions may be obtained by T- and $S L(2, Z)$ duality.

\section{$4 \quad D=5$ non-extreme solutions}

The extreme $D=5$ black holes with three charges can be obtained from the two different configurations [18]: three two-branes intersecting at a point $(2 \perp 2 \perp 2)$ and intersecting two-brane and five-brane along a common string $(2 \perp 5)$ with boost. We now briefly summarize the non-extreme solutions which correspond to $D=5$ black holes.

\subsection{Non-extreme intersecting $2 \perp 2 \perp 2$ M-branes}

For the intersecting $2 \perp 2 \perp 2$ M-branes, our metric is given by

$$
\begin{aligned}
d s_{11}^{2}= & \left(H_{1} H_{2} H_{3}\right)^{1 / 3}\left[-\left(H_{1} H_{2} H_{3}\right)^{-1} f d t^{2}+H_{1}^{-1}\left(d y_{1}^{2}+d y_{2}^{2}\right)+H_{2}^{-1}\left(d y_{3}^{2}+d y_{4}^{2}\right)\right. \\
& \left.+H_{3}^{-1}\left(d y_{5}^{2}+d y_{6}^{2}\right)+f^{-1} d r^{2}+r^{2} d \Omega_{3}^{2}\right] .
\end{aligned}
$$

In this section, our non-extreme deformation is parametrized by (3.2) with $\tilde{d}=2$.

We take our metrics and field strengths of the following forms:

$$
\begin{aligned}
H_{i} & =1+\frac{Q_{i}}{r^{2}}, \quad(i=1,2,3) \\
F_{012 r} & =\frac{1}{2} T_{1}^{\prime} ; \quad F_{034 r}=\frac{1}{2} T_{2}^{\prime} ; \quad F_{056 r}=\frac{1}{2} T_{3}{ }^{\prime}
\end{aligned}
$$


Substituting these into the field eqs. (2.1), we again find that there is a solution

$$
T_{i}=1-\frac{\sqrt{\left(Q_{i}+\mu_{1}\right)\left(Q_{i}+\mu_{2}\right)}}{r^{2}+Q_{i}}, \quad(i=1,2,3) .
$$

For $Q_{1}=Q_{2}=Q_{3}$ and $\mu_{2}=0$, this solution becomes the anisotropic six-brane solution in ref. [3]. For $\mu_{2}=0$ or under the replacement (3.5), this coincides with the solution in ref. [23].

If we use the parametrization (3.6), the nine-area at the outer horizon $r=\sqrt{\mu_{1}}$ is given by

$$
\begin{aligned}
A_{9} & =2 \pi^{2} L^{6}\left[r^{3}\left(H_{1} H_{2} H_{3}\right)^{1 / 2}\right]_{r=\mu_{1}^{1 / 2}}=2 \pi^{2} L^{6} \prod_{i=1}^{3} \sqrt{Q_{i}+\mu_{1}} \\
& =2 \pi^{2} L^{6}\left(\mu_{1}-\mu_{2}\right)^{3 / 2} \prod_{i=1}^{3} \cosh \delta_{i} .
\end{aligned}
$$

In the extreme limit $\mu_{1}, \mu_{2} \rightarrow \mu$ while keeping $\left(\mu_{1}-\mu_{2}\right)^{1 / 2} \cosh \delta_{i}$ finite, supersymmetry is recovered and the area becomes

$$
\left(A_{9}\right)_{B P S}=2 \pi^{2} L^{6} \sqrt{\left(Q_{1}+\mu\right)\left(Q_{2}+\mu\right)\left(Q_{3}+\mu\right)} .
$$

Upon toroidal compactification of $y_{1}, \cdots, y_{6}$, our solution reduces to the five-dimensional black hole with the Einstein-frame metric

$$
\begin{gathered}
d s_{5}^{2}=-\lambda^{2}(r) f(r) d t^{2}+\lambda^{-1}(r)\left[f^{-1}(r) d r^{2}+r^{2} d \Omega_{3}^{2}\right], \\
\lambda(r)=\left(H_{1} H_{2} H_{3}\right)^{-1 / 3}=\frac{r^{2}}{\left[\left(r^{2}+Q_{1}\right)\left(r^{2}+Q_{2}\right)\left(r^{2}+Q_{3}\right)\right]^{1 / 3}} .
\end{gathered}
$$

Under the replacement (3.5), this agrees with the metric of non-extreme five-dimensional black holes found in ref. [6, 15, 23], with three electric charges.

\subsection{Non-extreme intersecting $2 \perp 5$ M-branes with boost}

The non-extreme generalization of the supersymmetric configuration of a two-brane intersecting with a five-brane boosted along the common string [18] takes the form

$$
\begin{aligned}
d s_{11}^{2}= & H_{1}^{1 / 3} H_{2}^{2 / 3}\left[\left(H_{1} H_{2}\right)^{-1}\left(-K^{-1} f d t^{2}+K{\widehat{d y_{1}}}^{2}\right)+H_{1}^{-1} d y_{2}^{2}\right. \\
& \left.+H_{2}^{-1}\left(d y_{3}^{2}+\cdots+d y_{6}^{2}\right)+f^{-1} d r^{2}+r^{2} d \Omega_{3}^{2}\right]
\end{aligned}
$$


where

$$
\begin{aligned}
\widehat{d y_{1}} & =d y_{1}+\frac{\tilde{K}(r)}{K(r)} d t ; \quad F_{012 r}=\frac{1}{2} T_{1}^{\prime} ; \quad F_{2 a b c}=\frac{1}{2} \epsilon_{a b c r} T_{2}{ }^{\prime}, \\
K(r) & =1+\frac{P}{r^{2}} ; \quad H_{i}(r)=1+\frac{Q_{i}}{r^{2}},(i=1,2) .
\end{aligned}
$$

Here $a, b, c$ denote the angular coordinates of the transverse dimensions, and $f$ is the same as eq. (3.2). The charge $P$ corresponds to the momentum along the direction $y_{1}$.

Plugging these into the field eqs. (2.1), we find the solution

$$
\begin{aligned}
\tilde{K} & =-\frac{\sqrt{\left(P+\mu_{1}\right)\left(P+\mu_{2}\right)}}{r^{2}}, \\
T_{1} & =1-\frac{\sqrt{\left(Q_{1}+\mu_{1}\right)\left(Q_{1}+\mu_{2}\right)}}{r^{2}+Q_{1}} ; \quad T_{2}=1-\frac{\sqrt{\left(Q_{2}+\mu_{1}\right)\left(Q_{2}+\mu_{2}\right)}}{r^{2}} .
\end{aligned}
$$

If we use the parametrization (3.14), we again find that the nine-area at the outer horizon is given by

$$
\begin{aligned}
A_{9} & =2 \pi^{2} L^{6}\left[r^{3}\left(K H_{1} H_{2}\right)^{1 / 2}\right]_{r=\mu_{1}^{1 / 2}}=2 \pi^{2} L^{6} \sqrt{\left(Q_{1}+\mu_{1}\right)\left(Q_{2}+\mu_{1}\right)\left(P+\mu_{1}\right)} \\
& =2 \pi^{2} L^{6}\left(\mu_{1}-\mu_{2}\right)^{3 / 2} \cosh \delta_{1} \cosh \delta_{2} \cosh \gamma
\end{aligned}
$$

The extreme limit is again to send $\mu_{1}, \mu_{2} \rightarrow \mu$ while keeping $\left(\mu_{1}-\mu_{2}\right)^{1 / 2} \cosh \delta_{i}(\gamma)$ or $Q_{i}$ and $P$ finite. In this limit, the nine-area becomes

$$
\left(A_{9}\right)_{B P S}=2 \pi^{2} L^{6} \sqrt{\left(Q_{1}+\mu\right)\left(Q_{2}+\mu\right)(P+\mu)}
$$

The corresponding five-dimensional Einstein-frame metric is (4.6) with

$$
\lambda(r)=\left(H_{1} H_{2} K\right)^{-1 / 3}=\frac{r^{2}}{\left[\left(r^{2}+Q_{1}\right)\left(r^{2}+Q_{2}\right)\left(r^{2}+P\right)\right]^{1 / 3}},
$$

basically the metric found in ref. [6, 15, 23], with two electric and one magnetic charges.

\section{General analysis of $D \geq 6$ non-extreme solutions}

We have examined if the same method applies to higher dimensional case such as twobranes. One of our motivations for this analysis is that if we can have nonzero $\mu_{2}$, we 
can have extreme solutions with nonzero horizon area since then we need not set the non-extremality parameters to zero but to equal finite value.

It is certainly true that if we put $H_{2}=H_{3}=1$ in our intersecting $2 \perp 2 \perp 2 \mathrm{M}$ branes (4.1), this gives a special solution corresponding to a two-brane solution in elevendimensions, with additional backgrounds of field strengths $F_{034 r}$ and $F_{056 r}$. However, it turns out that these cannot be extended to higher dimensions unless $\mu_{2}=0$. Below we present a general analysis of the higher-dimensional solutions and show that the problem of solving field equations boils down to a simple algebraic algorithm.

We use this result to show that there is no extreme solutions with regular horizons of finite area except for special ones which are identified. It should be noted that our following analysis is not restrited to supersymmetric case in the extreme limit.

\section{$5.1 \quad$ Metrics}

Let us start the general analysis of solutions in higher dimensions. In order to see if there is any solution, it is sufficient to examine the case of equal charges. It is straightforward to generalize the results to solutions with different charges afterwards, as we will show in the next section. Hence we take the following metric as the most general static sphericallysymmetric one (boost charge will be considered later):

$$
d s_{11}^{2}=-H^{2 a_{0}} f d t^{2}+\sum_{\alpha=1}^{d-1} H^{2 a_{\alpha}} d y_{\alpha}^{2}+H^{2 b}\left(f^{-1} d r^{2}+r^{2} d \Omega_{\tilde{d}+1}^{2}\right),
$$

where $H=1+\frac{Q}{r^{\tilde{d}}}$ is a harmonic function in $(\tilde{d}+2)$ dimensions with the charge $Q, f$ is given in eq. (3.2), and $a$ 's and $b$ are constants to be determined.

A straightforward calculation using (2.5) yields

$$
\begin{aligned}
R_{00} & =H^{2\left(a_{0}-b\right)} f\left[-a_{0} \frac{\tilde{d}^{2} \tilde{Q}^{2}}{H^{2} r^{2 \tilde{d}+2}}+\left(a_{0}+1\right) \frac{\tilde{d}^{2} \mu_{1} \mu_{2}}{r^{2 \tilde{d}+2}}\right] \\
R_{\alpha \beta} & =H^{2\left(a_{\alpha}-b\right)}\left[a_{\alpha} \frac{\tilde{d}^{2} \tilde{Q}^{2}}{H^{2} r^{2 \tilde{d}+2}}-a_{\alpha} \frac{\tilde{d}^{2} \mu_{1} \mu_{2}}{r^{2 \tilde{d}+2}}\right] \delta_{\alpha \beta}, \quad(\alpha, \beta=1, \ldots, d-1), \\
R_{r r} & =\frac{1}{f}\left[a_{0} \frac{\tilde{d}^{2} \tilde{Q}^{2}}{H^{2} r^{2 \tilde{d}+2}}-\left(\sum_{\alpha=0}^{d-1} a_{\alpha}^{2}+a_{0}+\tilde{d} b^{2}-b\right) f \frac{\tilde{d}^{2} Q^{2}}{H^{2} r^{2 \tilde{d}+2}}-\left(a_{0}+1\right) \frac{\tilde{d}^{2} \mu_{1} \mu_{2}}{r^{2 \tilde{d}+2}}\right] \\
R_{a b} & =\left[b \frac{\tilde{d}^{2} \tilde{Q}^{2}}{H^{2} r^{2 \tilde{d}+2}}-\tilde{d}(\tilde{d} b-1) \frac{\mu_{1} \mu_{2}}{r^{2 \tilde{d}+2}}\right] g_{a b},
\end{aligned}
$$


where we have defined $\tilde{Q}^{2}=\left(Q+\mu_{1}\right)\left(Q+\mu_{2}\right)$. In deriving the result (5.2), we have imposed the condition

$$
\sum_{\alpha=0}^{d-1} a_{\alpha}+\tilde{d} b=0
$$

Unless this condition is obeyed, there remain quite complicated terms which cannot satisfy field equations. Also this is satisfied by all known solutions.

From the field eq. (2.1), we have

$$
\left(F^{2}\right)_{M N}=3 R_{M N}+3 R g_{M N}
$$

which, together with (5.2), yields

$$
\begin{aligned}
\left(F^{2}\right)_{a b}= & {\left[3\left(a_{0}+2 b\right) \frac{\tilde{d}^{2} \tilde{Q}^{2}}{H^{2} r^{2 \tilde{d}+2}}-3\left(\sum_{\alpha=0}^{d-1} a_{\alpha}^{2}+a_{0}+\tilde{d} b^{2}-b\right) f \frac{\tilde{d}^{2} Q^{2}}{H^{2} r^{2 \tilde{d}+2}}\right.} \\
& \left.-3 \tilde{d}\left\{\tilde{d}\left(a_{0}+2 b+1\right)-2\right\} \frac{\mu_{1} \mu_{2}}{r^{2 \tilde{d}+2}}\right] g_{a b} .
\end{aligned}
$$

Up to this point, we have made no assumption on dimensionality. From now on, we specialize to $D \geq 6(\tilde{d} \geq 3)$. There are two cases to be discussed separately.

\subsection{The case of vanishing $\left(F^{2}\right)_{a b}$}

Let us first consider the case in which $\left(F^{2}\right)_{a b}$ vanishes. This is always the case for $D \geq 7(\tilde{d} \geq 4)$ since we have only fourth-rank antisymmetric tensor. From the first two terms in eq. (5.5), we must have

$$
b=-\frac{1}{2} a_{0} ; \quad \sum_{\alpha=1}^{d-1} a_{\alpha}^{2}=-\frac{\tilde{d}+4}{4} a_{0}^{2}-\frac{3}{2} a_{0},
$$

which gives

$$
\left(F^{2}\right)_{a b}=-3 \tilde{d}(\tilde{d}-2) \frac{\mu_{1} \mu_{2}}{r^{2 \tilde{d}+2}} g_{a b} .
$$

This does not vanish for $\tilde{d} \geq 3$ unless $\mu_{2}=0$, implying that (5.6) is valid and $\mu_{2}$ must vanish.

With the condition (5.6) and $\mu_{2}=0$, we find from (5.2) and (5.4)

$$
\left(F^{2}\right)_{00}=-H^{3 a_{0}} f \frac{9}{2} a_{0} \frac{\tilde{d}^{2} \tilde{Q}^{2}}{H^{2} r^{2 \tilde{d}+2}}
$$




$$
\begin{aligned}
\left(F^{2}\right)_{\alpha \alpha} & =H^{\left(2 a_{\alpha}+a_{0}\right)} \frac{3}{2}\left(2 a_{\alpha}+a_{0}\right) \frac{\tilde{d}^{2} \tilde{Q}^{2}}{H^{2} r^{2 \tilde{d}+2}} \\
\left(F^{2}\right)_{r r} & =\frac{1}{f} \frac{9}{2} a_{0} \frac{\tilde{d}^{2} \tilde{Q}^{2}}{H^{2} r^{2 \tilde{d}+2}} .
\end{aligned}
$$

So far, we have made no assumption on the background field strengths. In order to satisfy the field equations, let us introduce all possible terms for the field strengthst

$$
F_{0 \alpha \beta r}=\frac{1}{2} a_{\alpha \beta} T_{1}^{\prime}
$$

where $a_{\alpha \beta}$ are constants antisymmetric in their indices. Equating the contribution from (5.9) to (5.8), we obtain

$$
T_{1}^{\prime}=H^{a_{0}+a_{\alpha}+a_{\beta}-1} \frac{\tilde{d} \tilde{Q}}{r^{\tilde{d}+1}}
$$

and

$$
\begin{aligned}
\sum_{\alpha<\beta} a_{\alpha \beta}^{2} & =-3 a_{0}, \\
\sum_{\beta(\neq \alpha)} a_{\alpha \beta}^{2} & =-\left(2 a_{\alpha}+a_{0}\right), \text { for } \alpha=1, \ldots, d-1 .
\end{aligned}
$$

Consistency with the field eq. (2.1) requires that only the coefficients $a_{\alpha \beta}$ that satisfy

$$
a_{0}+a_{\alpha}+a_{\beta}=-1
$$

can be nonvanishing. In this case eq. (5.10) gives

$$
T_{1}=1-\frac{\tilde{Q}}{r^{\tilde{d}}+Q} .
$$

Thus our problem of solving field equations boils down to the simple algebraic one of finding solutions to (5.3), (5.6) and (5.11). Simple solutions are

$$
\begin{aligned}
\text { Two - brane : } & a_{0}=a_{1}=a_{2}=-\frac{1}{3} ; a_{3}=\cdots=a_{d-1}=b=\frac{1}{6} ; a_{12}=1 . \\
2 \perp 2: & a_{0}=-\frac{2}{3} ; a_{1}=a_{2}=a_{3}=a_{4}=-\frac{1}{6} ; a_{5}=\cdots=a_{d-1}=b=\frac{1}{3} ; \\
& a_{12}=a_{34}=1,(\text { others }=0) .
\end{aligned}
$$

\footnotetext{
${ }^{4}$ We are not considering the case in which KK monopoles exist [31]. It is easy to see that this does not affect our main results.
} 
If there are monopole backgrounds, the last condition (5.11) is slightly changed, but the general properties of the solutions remain the same.

These results are enough to establish that there are no solutions with regular horizons of finite area with just one exception. From the condition (5.6), one finds

$$
0>a_{0}>-\frac{6}{\tilde{d}+4} .
$$

On the other hand, the nine-area is given by

$$
A_{9}=\omega_{\tilde{d}+1} L^{d-1}\left[r^{\tilde{d}+1} H^{\sum_{\alpha=1}^{5} a_{\alpha}+(\tilde{d}+1) b}\right]_{r=\mu_{1}^{1 / \tilde{d}}},
$$

which, with help of eqs. (5.3) and (5.6), is cast into

$$
\begin{aligned}
A_{9} & =\omega_{\tilde{d}+1} L^{d-1}\left[r^{\tilde{d}+1} H^{-3 a_{0} / 2}\right]_{r=\mu_{1}^{1 / \tilde{d}}}, \\
& \rightarrow \omega_{\tilde{d}+1} L^{d-1} \mu_{1}^{(\tilde{d}+1) / \tilde{d}+3 a_{0} / 2} Q^{-3 a_{0} / 2}
\end{aligned}
$$

near the extreme limit $\mu_{1} \sim 0$. Here $\omega_{\tilde{d}+1}$ is the volume of the unit $(\tilde{d}+1)$-sphere

$$
\omega_{\tilde{d}+1}=\frac{2 \pi^{\frac{\tilde{d}}{2}+1}}{\Gamma\left(\frac{\tilde{d}}{2}+1\right)} .
$$

However, the area (5.18) vanishes for (5.16) in the extreme limit.

If we can incorporate boost, it introduces the factor $K^{1 / 2}$ which has the effect of reducing the exponent of $\mu_{1}$ in (5.18) by $1 / 2$. This means that we have the possibility of nonvanishing nine-area for $a_{0}=-(\tilde{d}+2) / 3 \tilde{d}$. To have the boost, we must have a null isometry in another direction, say $y_{1}$ [27]. Thus we must have

$$
a_{0}=a_{1}=-\frac{\tilde{d}+2}{3 \tilde{d}} .
$$

Substituting (5.20) back into the second condition in (5.6) shows that there is a unique solution for $\tilde{d}=4$ :

$$
\begin{aligned}
& a_{0}=a_{1}=-\frac{1}{2} ; \quad a_{2}=a_{3}=a_{4}=0 ; \quad b=\frac{1}{4} ; \\
& a_{1 \alpha}=\frac{1}{\sqrt{2}},(\alpha=2,3,4),
\end{aligned}
$$

where use has also been made of the conditions (5.11) in deriving $a_{1 \alpha}$. The field strengths $F_{01 \alpha r}(\alpha=2,3,4)$ are nonvanishing and provide sources for 2 -branes lying in the planes 
$(1, \alpha)$. We thus see that this configuration corresponds to three 2-branes intersecting over a string $\left(2^{3}\right)$. This is one of the two cases in which the nine-area does not vanish in the extreme limit. (The other case will be given in the next subsection.) For all other cases, the nine-area always vanishes in the limit. This establishes the promised result.

\subsection{The case of nonvanishing $\left(F^{2}\right)_{a b}$}

Let us turn to the case of nonvanishing $\left(F^{2}\right)_{a b}$. This is possible only for $\tilde{d}=3$, since we can then introduce

$$
F_{a b c d}=\frac{1}{2} \epsilon_{a b c d r} T_{2}^{\prime}
$$

where the Bianchi identity in eq. (2.1) requires that $T_{2}$ should be a harmonic function. This gives

$$
\left(F^{2}\right)_{a b}=\frac{3}{2}\left(T_{2}^{\prime}\right)^{2} H^{-6 b} g_{a b}
$$

Equating this with the first term in eq. (5.5), we must have

$$
b=\frac{1}{3} ; \quad \sum_{\alpha=0}^{5} a_{\alpha}^{2}=-a_{0}-3 b^{2}+b ; \quad 3\left(a_{0}+2 b+1\right)=2,
$$

in order to have nonvanishing $\mu_{2}$. However, this leads to

$$
\left(F^{2}\right)_{a b}=-9 \frac{\tilde{Q}^{2}}{H^{2} r^{8}} g_{a b}
$$

which is negative and cannot be consistent with eq. (5.23). This means that we must have $\mu_{2}=0$. Similar reasoning excludes the possibility of equating (5.23) with other terms in (5.5) unless $\mu_{2}=0$. We thus conclude that $\mu_{2}$ must again be zero.

Putting $\mu_{2}=0$, we learn that either the first term or second term in (5.5) must balance with the contribution from the field strengths (5.23). It is easy to exclude the second possibility by an analysis similar to the above. So we are left with the first possibility:

$$
b=\frac{1}{3} ; \quad \sum_{\alpha=1}^{5} a_{\alpha}^{2}=-a_{0}\left(a_{0}+1\right) .
$$

\footnotetext{
${ }^{5}$ The solutions with monopoles discussed in ref. [31] suggest that there is no other case with nonzero area.
} 
We then have the solution for this part

$$
T_{2}=\sqrt{\frac{2}{3}\left(3 a_{0}+2\right)}\left(1-\frac{\tilde{Q}}{r^{3}}\right) .
$$

With the condition (5.26), we find from (5.2) and (5.4)

$$
\begin{aligned}
\left(F^{2}\right)_{00} & =-H^{2\left(a_{0}-1 / 3\right)} f\left(6 a_{0}+1\right) \frac{9 \tilde{Q}^{2}}{H^{2} r^{8}} \\
\left(F^{2}\right)_{\alpha \alpha} & =H^{2\left(a_{\alpha}-1 / 3\right)}\left(3 a_{\alpha}+3 a_{0}+1\right) \frac{9 \tilde{Q}^{2}}{H^{2} r^{8}}, \\
\left(F^{2}\right)_{r r} & =\frac{1}{f}\left(6 a_{0}+1\right) \frac{9 \tilde{Q}^{2}}{H^{2} r^{8}} .
\end{aligned}
$$

As our field strengths, we introduce (5.9). The same analysis as above shows that $T_{1}$ is given by eq. (5.13) with $\tilde{d}=3$ and

$$
\begin{aligned}
\sum_{\alpha<\beta} a_{\alpha \beta}^{2} & =-\frac{2}{3}\left(6 a_{0}+1\right), \\
\sum_{\beta(\neq \alpha)} a_{\alpha \beta}^{2} & =-\frac{2}{3}\left(3 a_{\alpha}+3 a_{0}+1\right), \text { for } \alpha=1, \ldots, 5,
\end{aligned}
$$

where only those $a_{\alpha \beta}$ are nonvanishing when (5.12) is satisfied.

Thus our problem again reduces to the algebraic one of finding solutions to (5.3), (5.12), (5.26) and (5.29). Simple examples of the solutions are

$$
\begin{aligned}
& \text { Five }- \text { brane : } a_{0}=a_{1}=\cdots=a_{5}=-\frac{1}{6} ; a_{\alpha \beta}=0 . \\
& 2 \subset 5: \quad a_{0}=a_{1}=a_{2}=-\frac{1}{3} ; \quad a_{12}=\sqrt{\frac{2}{3}} ; \quad(\text { others }=0) .
\end{aligned}
$$

These examples with boost will be summarized in the next section.

Let us finally examine if we can have solutions with horizons of finite area in the extreme limit. From the reality of (5.27) and (5.26), $a_{0}$ is restricted to be

$$
0>a_{0}>-\frac{2}{3} \text {. }
$$

On the other hand, with the use of eq. (5.3), the nine-area is given by

$$
A_{9}=\omega_{4} L^{5}\left[r^{4} H^{b-a_{0}}\right]_{r=\mu_{1}^{1 / 3}} \rightarrow \omega_{4} L^{5} \mu_{1}^{1+a_{0}} Q^{-a_{0}+1 / 3},
$$


near the extreme limit. This vanishes for (5.32) in the extreme limit.

What about the possibility of introducing boost? Since this has the effect of reducing the exponent in (5.33) by $1 / 2$, we have the possibility of getting nonvanishing nine-area for $a_{0}=-\frac{1}{2}$. Choosing $y_{1}$ for the direction of the null isometry, we again find a unique solution

$$
\begin{aligned}
& a_{0}=a_{1}=-\frac{1}{2} ; \quad a_{2}=\cdots=a_{5}=0, \\
& a_{1 \alpha}=\frac{1}{\sqrt{3}},(\alpha=2, \ldots, 5) ; \text { other } a^{\prime} \mathrm{s}=0,
\end{aligned}
$$

where use has been made of the conditions (5.26), (5.29) and (5.12). This is similar to the solution (5.21) and allows the interpretation of four 2-branes and a 5-brane intersecting over a string $\left(2^{4} \subset 5\right)$. These two solutions (5.21) and (5.34) constitute the only cases in which the nine-area does not vanish in the extreme limit.

We thus conclude that $\mu_{2}=0$ necessarily in higher dimensions and that the ninearea must vanish in the extreme limit except for the two special cases of seven- and six-dimensional ones (5.21) and (5.34).

\section{$6 \quad D \geq 6$ non-extreme solutions}

In this section, we summarize typical solutions for higher dimensions for completeness, including the interesting case of the intersecting two-branes with regular horizons of finite area. Though some of them are known ones [3, 23], it is instructive to see how the solutions with equal charges considered in the previous section are generalized and also to see how these are consistent with the previous analysis.

In what follows, we take $\mu_{2}=0$ and

$$
\begin{aligned}
& \widehat{d y_{1}}=d y_{1}+\frac{\tilde{K}(r)}{K(r)} d t ; \quad \tilde{Q}^{2}=Q\left(Q+\mu_{1}\right) ; \quad \tilde{P}^{2}=P\left(P+\mu_{1}\right), \\
& f(r)=1-\frac{\mu_{1}}{r^{\tilde{d}}} ; \quad H(r)=1+\frac{Q}{r^{\tilde{d}}} ; \quad K(r)=1+\frac{P}{r^{\tilde{d}}} ; \quad \tilde{K}=-\frac{\tilde{P}}{r^{\tilde{d}}} .
\end{aligned}
$$




\section{1 $2^{3}$ with boost in $D=7$}

The boosted solution (5.21) identified to have nonvanishing area has the metric

$$
\begin{aligned}
d s_{11}^{2}= & H^{1 / 2}\left[H^{-3 / 2}\left(-K^{-1} f d t^{2}+K{\widehat{d y_{1}}}^{2}\right)+H^{-1 / 2}\left(d y_{2}^{2}+d y_{3}^{2}+d y_{4}^{2}\right)\right. \\
& \left.+f^{-1} d r^{2}+r^{2} d \Omega_{5}^{2}\right],
\end{aligned}
$$

for $\tilde{d}=4$. It does not seem possible to have different charges in this case. The solution is

$$
F_{01 \alpha r}=\frac{1}{2 \sqrt{2}} T_{1}^{\prime},(\alpha=2,3,4) ; \quad T_{1}=1-\frac{\tilde{Q}}{r^{4}+Q},
$$

The nine-area is

$$
A_{9}=\omega_{5} L^{4}\left(Q+\mu_{1}\right)^{3 / 4}\left(P+\mu_{1}\right)^{1 / 2},
$$

which does not vanish in the "extreme" limit $\mu_{1} \rightarrow 0$, as anticipated. However, examining the condition of supersymmetry (2.2), one finds that no supersymmetry is recovered in the limit $\mu_{1} \rightarrow 0$. So it is true that this is a solution of the field equations, but this limit simply implies that the outer and inner horizons at $r=\mu_{1}^{1 / 4}, 0$ become degenerate. In particular, it may not be easy to control quantum corrections even near the limit.

The corresponding $(\tilde{d}+3)$-dimensional Einstein-frame metric is (here $\tilde{d}=4)$ :

$$
\begin{gathered}
d s_{\tilde{d}+3}^{2}=-\lambda^{\tilde{d}}(r) f(r) d t^{2}+\lambda^{-1}(r)\left[f^{-1}(r) d r^{2}+r^{2} d \Omega_{\tilde{d}+1}^{2}\right], \\
\lambda(r)=H^{-3 / 10} K^{-1 / 5}=\frac{r^{2}}{\left(r^{4}+Q\right)^{3 / 10}\left(r^{3}+P\right)^{1 / 5}} .
\end{gathered}
$$

\section{2 $2^{4} \subset 5$ with boost in $D=6$}

The boosted solution (5.34) with nonvanishing area has the metric

$$
\begin{aligned}
d s_{11}^{2}= & H^{2 / 3}\left[H^{-5 / 3}\left(-K^{-1} f d t^{2}+K{\widehat{d y_{1}}}^{2}\right)+H^{-2 / 3}\left(d y_{2}^{2}+\cdots+d y_{5}^{2}\right)\right. \\
& \left.+f^{-1} d r^{2}+r^{2} d \Omega_{4}^{2}\right]
\end{aligned}
$$

with $\tilde{d}=3$. It does not seem possible to have different charges in this case either. The solution is

$$
\begin{aligned}
& F_{01 \alpha r}=\frac{1}{2 \sqrt{3}} T_{1}^{\prime}, \quad(\alpha=2, \ldots, 5) ; \quad F_{a b c d}=\frac{1}{2} T_{2}{ }^{\prime}, \\
& T_{1}=1-\frac{\tilde{Q}}{r^{3}+Q} ; \quad T_{2}=\frac{1}{\sqrt{3}}\left(1-\frac{\tilde{Q}}{r^{3}}\right),
\end{aligned}
$$


The nine-area is

$$
A_{9}=\omega_{4} L^{5}\left(Q+\mu_{1}\right)^{5 / 6}\left(P+\mu_{1}\right)^{1 / 2},
$$

which again does not vanish in the "extreme" limit but supersymmetry is not recovered.

The corresponding six-dimensional Einstein-frame metric is (6.5) with $\tilde{d}=3$ and

$$
\lambda(r)=H^{-5 / 12} K^{-1 / 4}=\frac{r^{2}}{\left(r^{3}+Q\right)^{5 / 12}\left(r^{3}+P\right)^{1 / 4}} .
$$

\section{3 $2 \subset 5$ with boost in $D=6$}

The solution (5.31) with boost has the metric

$$
\begin{aligned}
d s_{11}^{2}= & H^{2 / 3}\left[H^{-4 / 3}\left(-K^{-1} f d t^{2}+K{\widehat{d y_{1}}}^{2}+d y_{2}^{2}\right)+H^{-2 / 3}\left(d y_{3}^{2}+\cdots+d y_{5}^{2}\right)\right. \\
& \left.+f^{-1} d r^{2}+r^{2} d \Omega_{4}^{2}\right],
\end{aligned}
$$

with $\tilde{d}=3$. The solution is

$$
\begin{aligned}
& F_{012 r}=\frac{1}{2} T_{1}{ }^{\prime} ; \quad F_{a b c d}=\frac{1}{2} T_{2}{ }^{\prime}, \\
& T_{1}=1-\frac{\tilde{Q}}{r^{3}+Q} ; \quad T_{2}=\sqrt{\frac{2}{3}}\left(1-\frac{\tilde{Q}}{r^{3}}\right) .
\end{aligned}
$$

The nine-area is

$$
A_{9}=\omega_{4} L^{5} \mu_{1}^{1 / 6}\left(Q+\mu_{1}\right)^{2 / 3}\left(P+\mu_{1}\right)^{1 / 2},
$$

which vanishes in the "extreme" limit but supersymmetry remains broken.

The corresponding six-dimensional Einstein-frame metric is (6.5) with $\tilde{d}=3$ and

$$
\lambda(r)=H^{-1 / 3} K^{-1 / 4}=\frac{r^{7 / 4}}{\left(r^{3}+Q\right)^{1 / 3}\left(r^{3}+P\right)^{1 / 4}} .
$$

\subsection{Non-extreme two-brane with boost}

It is possible to discuss boosted versions of $6 \leq D(\equiv \tilde{d}+3) \leq 9$ two-brane solutions (5.14) together. We take our metric as

$$
\begin{aligned}
d s_{11}^{2}= & H^{1 / 3}\left[H^{-1}\left(-K^{-1} f d t^{2}+K{\widehat{d y_{1}}}^{2}+d y_{2}^{2}\right)+d y_{3}^{2}+\cdots+d y_{d-1}^{2}\right. \\
& \left.+f^{-1} d r^{2}+r^{2} d \Omega_{\tilde{d}+1}^{2}\right] .
\end{aligned}
$$


We find that the solution to the field equation (2.1) is given by [23]

$$
F_{012 r}=\frac{1}{2} T^{\prime} ; \quad T=1-\frac{\tilde{Q}}{r^{\tilde{d}}+Q},
$$

The nine-area at the outer horizon $r=\mu_{1}^{1 / \tilde{d}}$ is given by

$$
\begin{aligned}
A_{9} & =\omega_{\tilde{d}+1} L^{d-1}\left[r^{\tilde{d}+1}(K H)^{1 / 2}\right]_{r=\mu_{1}^{1 / \tilde{d}}} \\
& =\omega_{\tilde{d}+1} L^{d-1} \mu_{1}^{1 / \tilde{d}} \sqrt{\left(Q+\mu_{1}\right)\left(P+\mu_{1}\right)} .
\end{aligned}
$$

In the extreme limit $\mu_{1} \rightarrow 0$, this vanishes [23, 22]. Here and in all the following examples, supersymmetry is recovered in the extreme limit.

The corresponding $(\tilde{d}+3)$-dimensional Einstein-frame metric is (6.5) with

$$
\lambda(r)=(H K)^{-1 /(\tilde{d}+1)}=\frac{r^{2 \tilde{d} /(\tilde{d}+1)}}{\left[\left(r^{\tilde{d}}+Q\right)\left(r^{\tilde{d}}+P\right)\right]^{1 /(\tilde{d}+1)}} .
$$

\subsection{Non-extreme five-brane with boost}

The non-extreme generalization of the a five-brane (5.30) with a boost has the metrics

$$
d s_{11}^{2}=H^{2 / 3}\left[H^{-1}\left(-K^{-1} f d t^{2}+K{\widehat{d y_{1}}}^{2}+d y_{2}^{2}+\cdots+d y_{5}^{2}\right)+f^{-1} d r^{2}+r^{2} d \Omega_{4}^{2}\right],
$$

with $\tilde{d}=3$. We find

$$
F_{a b c d}=\frac{1}{2} T^{\prime} ; \quad T=1-\frac{\tilde{Q}}{r^{3}} .
$$

again solve the field equations (2.1) [23].

A similar analysis to the previous subsection shows that there is a regular horizon at $r=\mu_{1}^{1 / 3}$ and that the nine-area vanishes in the extreme limit. The expressions for nine-area and resulting five-dimensional metrics are similar to (6.17) $-(6.18)$ with $\tilde{d}=3$.

\subsection{Intersecting $2 \perp 2$ M-branes}

The final two-charge versions of higher-dimensional black hole solutions we give explicitly are the intersecting $2 \perp 2$ solutions (5.15). These exist in $D=6,7$ dimensions and we 
discuss them together. The metric is

$$
\begin{aligned}
d s_{11}^{2}= & \left(H_{1} H_{2}\right)^{1 / 3}\left[-\left(H_{1} H_{2}\right)^{-1} f d t^{2}+H_{1}^{-1}\left(d y_{1}^{2}+d y_{2}^{2}\right)+H_{2}^{-1}\left(d y_{3}^{2}+d y_{4}^{2}\right)+d y_{d-1}^{2}\right. \\
& \left.+f^{-1} d r^{2}+r^{2} d \Omega_{\tilde{d}+1}^{2}\right]
\end{aligned}
$$

where $d=6$ or $5(D=\tilde{d}+3=6$ or 7 , respectively). The solution is

$$
F_{012 r}=\frac{1}{2} T_{1}^{\prime} ; \quad F_{034 r}=\frac{1}{2} T_{2}^{\prime} ; \quad T_{i}=1-\frac{\tilde{Q}_{i}}{r^{\tilde{d}}+Q_{i}},(i=1,2),
$$

in an obvious notation.

The nine-area at the outer horizon $r=\mu_{1}^{1 / \tilde{d}}$ is given by

$$
\begin{aligned}
A_{9} & =\omega_{\tilde{d}+1} L^{d-1}\left[r^{\tilde{d}+1}\left(H_{1} H_{2}\right)^{1 / 2}\right]_{r=\mu_{1}^{1 / \tilde{d}}} \\
& =\omega_{\tilde{d}+1} L^{d-1} \mu_{1}^{1 / \tilde{d}} \sqrt{\left(Q_{1}+\mu_{1}\right)\left(Q_{2}+\mu_{1}\right)}
\end{aligned}
$$

which vanishes in the extreme limit $\mu_{1} \rightarrow 0$. The corresponding $(\tilde{d}+3)$-dimensional Einstein-frame metric is (6.5) with $\lambda(r)$ almost the same as (6.18).

\section{ADM mass and entropy}

Upon dimensional reduction of the world-volumes $\left((d-1)\right.$-dimensional $y_{\alpha}$ space $)$ of the intersecting M-branes, we obtain the black hole solutions in $\tilde{d}+3$ dimensions. From eqs. (3.9), (3.10), (3.17), (4.6), (4.7), (4.13) and (6.5), we see that, except for the special cases in (6.6), (6.10) and (6.14), the Einstein-frame metrics can be written universally as

$$
d s_{\tilde{d}+3}^{2}=-\lambda^{\tilde{d}}(r) f(r) d t^{2}+\lambda^{-1}(r)\left[f^{-1}(r) d r^{2}+r^{2} d \Omega_{\tilde{d}+1}^{2}\right],
$$

where

$$
\lambda(r)=\left(H_{1} H_{2} \cdots H_{n}\right)^{-1 /(\tilde{d}+1)} ; \quad H_{i}=1+\frac{Q_{i}}{r^{\tilde{d}}}, \quad(i=1, \ldots, n),
$$

$f$ is given in (3.2) and we have expressed all charges by $Q_{i}$. For $\tilde{d} \geq 3$, we should put $\mu_{2}=0$.

From the asymptotic form of the metric $g_{00}$, we can read off the ADM mass [28]:

$$
M_{A D M}=a\left[(\tilde{d}+1)\left(\mu_{1}+\mu_{2}\right)+\tilde{d} \sum_{i=1}^{n} Q_{i}\right]
$$


where the constant $a$ is defined by

$$
a=\frac{\omega_{\tilde{d}+1}}{2 \kappa^{2}} L^{d-1},
$$

and we have defined the eleven-dimensional Newton's constant as $G_{11}=\kappa^{2} / 8 \pi$. For the solutions (6.6) $(\tilde{d}=4)$ and (6.10) $(\tilde{d}=3)$,

$$
M_{A D M}^{e x c}=a\left[(\tilde{d}+1) \mu_{1}+(\tilde{d}+2) Q+\tilde{d} P\right]
$$

For (6.14), we find

$$
M_{A D M}^{e x c}=a\left[4 \mu_{1}+4 Q+3 P\right],
$$

If we use, instead of $Q_{i}$, the charges

$$
P_{i} \equiv \frac{\mu_{1}-\mu_{2}}{2} \sinh 2 \delta_{i}
$$

which are also fixed in the extreme limit, the ADM mass (7.3) is cast into the form

$$
M_{A D M}=a \tilde{d}\left[\sum_{i=1}^{n} \sqrt{P_{i}^{2}+\left(\frac{\mu_{1}-\mu_{2}}{2}\right)^{2}}+\lambda\left(\mu_{1}+\mu_{2}\right)\right],
$$

where the constant $\lambda$ is defined by

$$
\lambda \equiv \frac{\tilde{d}+1}{\tilde{d}}-\frac{n}{2} .
$$

This constant $\lambda$ vanishes in our solutions for $D=4,(n=4)$ and $D=5,(n=3)$ in sects. 3, $4 ; n=1$ and $\lambda=\frac{\tilde{d}+2}{2 \tilde{d}}$ for two- and five-branes without boost in sect. 6 ; for other usual solutions in higher dimensions, $n=2$ and $\lambda=\frac{1}{d}$. If we try to write (7.5) as (7.8), it takes the form

$$
M_{A D M}^{e x c}=a\left[\tilde{d} \sqrt{P_{1}^{2}+\left(\frac{\mu_{1}}{2}\right)^{2}}+(\tilde{d}+2) \sqrt{P_{2}^{2}+\left(\frac{\mu_{1}}{2}\right)^{2}}\right],
$$

in an obvious notation and an "effective" constant $\lambda$ is zero. Similarly, for (7.6), one finds that the effective $\lambda=\frac{1}{6}$.

The constant $\lambda$ gives the measure how the area or entropy vanish in the extreme limit: If it is positive, they vanish like $\sim \mu_{1}^{\lambda}$; if it is zero, they remain finite. 
The entropy is given by

$$
\begin{aligned}
S_{B H} & =\frac{2 \pi A_{9}}{\kappa^{2}}=4 \pi a \mu_{1}^{\lambda} \prod_{i=1}^{n}\left[\left(\mu_{1}-\mu_{2}\right)^{1 / 2} \cosh \delta_{i}\right] \\
& =4 \pi a\left(\mu_{1}-\mu_{2}\right)^{\frac{\tilde{d}+1}{d}-\lambda} \mu_{1}^{\lambda} \prod_{i=1}^{n} \cosh \delta_{i} .
\end{aligned}
$$

This agrees with the similar expression in ref. [23]. Again for (6.2) and (6.7), one has

$$
S_{B H}^{e x c}=\frac{2 \pi A_{9}}{\kappa^{2}}=4 \pi a \mu_{1}^{(\tilde{d}+1) / \tilde{d}}\left(\cosh \delta_{1}\right)^{(\tilde{d}+2) / \tilde{d}} \cosh \delta_{2} .
$$

Let us next compute the Hawking temperature from the periodicity of the Euclideanized geometry. Suppose that our metric is of the form

$$
d s^{2}=e^{2 u} d \tau^{2}+e^{2 v} d r^{2}+r^{2} d \Omega_{\tilde{d}+1}^{2},
$$

and $e^{2 u}=0$ at the horizon $r=r_{0}$. Then near the horizon, the metric (7.13) can be written as

$$
d s^{2}=\left[\left.\left(e^{u}\right)^{\prime} e^{-v}\right|_{r=r_{0}}\right]^{2} R^{2} d \tau^{2}+d R^{2}+r^{2} d \Omega_{\tilde{d}+1}^{2},
$$

where $R=0$ at $r=r_{0}$ and $r$ should be regarded as a function of $R$. We see that $\tau$ can be interpreted as angular coordinate on the $R-\tau$ plane. From the condition that there is no singularity at $R=0$, we find that $\tau$ should be a periodic variable with period $\beta=1 / T_{H}$ with

$$
2 \pi T_{H}=\left.\left(e^{u}\right)^{\prime} e^{-v}\right|_{r=r_{0}} .
$$

This formula gives the Hawking temperature for our normal solutions

$$
T_{H}=\frac{\tilde{d}}{4 \pi}\left(\mu_{1}-\mu_{2}\right)^{\lambda-1 / \tilde{d}} \mu_{1}^{-\lambda} \prod_{i=1}^{n}\left(\cosh \delta_{i}\right)^{-1},
$$

and the entropy (7.11) can be written as

$$
S_{B H}=a \tilde{d} \frac{\mu_{1}-\mu_{2}}{T_{H}} .
$$

Expressed in terms of the charges and $\mu_{1}, \mu_{2}$, the entropy can be transformed into

$$
\begin{aligned}
S_{B H} & =4 \pi a \mu_{1}^{\lambda} \prod_{i=1}^{n} \sqrt{Q_{i}+\mu_{1}} \\
& =4 \pi a \mu_{1}^{\lambda} \prod_{i=1}^{n}\left[\sqrt{P_{i}^{2}+\left(\frac{\mu_{1}-\mu_{2}}{2}\right)^{2}}+\frac{\mu_{1}-\mu_{2}}{2}\right]^{1 / 2} .
\end{aligned}
$$


For the exceptional cases, the powers of the charges in the product change, but the power of $\mu_{1}$ in front remains the same.

The extreme limit is to send $\mu_{1} \rightarrow \mu_{2}(=0$ for $D \geq 6)$ with $\left(\mu_{1}-\mu_{2}\right)^{1 / 2} \cosh \delta_{i}$ fixed. In this limit, the entropy (7.11) for $\lambda=0$ and (7.12) is nonvanishing but the Hawking temperature vanishes in our all solutions.

Finally we note that the ADM mass $M_{A D M}$ in our solutions in $D=4,5$ dimensions in sects. 3 and 4 resembles the energy of a system of relativistic particles with masses $P_{i}$ and momenta proportional to $\left(\mu_{1}-\mu_{2}\right)$. For other higher dimensional solutions, the ADM mass does not have such an interpretation.

\section{Conclusions}

We have presented non-extreme solutions in the form with two nonvanishing deformation parameters, similar to the four-dimensional Reissner-Nordstrøm black holes. They turn out to be equivalent to those extensively studied in ref. [23] under the replacement (3.5) for $D=4$ and 5 dimensions.

We have also tried to find higher-dimensional solutions. We have carried out a rather systematic analysis of the solutions in the case of equal charges and reduced the problem to a simple algebraic one. In the process, we were able to show that the generalization of these solutions with two nonvanishing deformation parameters is impossible, and that the area of the black holes in general vanishes in the extreme limit for static sphericallysymmetric ones in higher dimensions. The only exceptions are the solutions (6.6) in $D=7$ and (6.10) in $D=6$ dimensions. Unfortunately supersymmetry remains broken in the limit even though the inner and outer horizons coincide and the Hawking temperature vanishes. If we insist that supersymmetry should be recovered in the extreme limit, these exceptional solutions may not be regarded as "good" solutions. Together with the results in refs. [6, 23], our results imply that the extreme solutions in $D \geq 6$ necessarily involve naked singularities or do not have regular horizon of finite area except for (6.6) and (6.10) as long as we consider static spherically-symmetric solutions.

It would be quite interesting to give a statistical explanation of the entropy [8$16,21,22,28,29]$ and examine the Hawking radiation for these solutions, in order to gain 
insight into the quantum M-theory and also to understand what new features emerge from this kind of investigation. Our algebraic results should also be useful for finding new solutions. We hope to discuss these questions elsewhere.

\section{Acknowledgements}

We would like to thank R. Emparan, I. Klebanov and A. Tseytlin for pointing out the relations of our solutions in $D=4,5$ to those in refs. [18, 22, 23] by the redefinition (3.5). Special thanks are due to A. Tseytlin for useful correspondence which helped us to improve the manuscript. 


\section{References}

[1] A. Dabholkar, G. Gibbons, J. A. Harvey and F. Ruiz Ruiz, Nucl. Phys. B340 (1990) 33; for a review and earlier references, see M. Duff, R. Khuri and J. X. Lu, Phys. Rep. 259 (1995) 213, hep-th/9412184.

[2] G. T. Horowitz and A. Strominger, Nucl. Phys. B360 (1991) 197.

[3] R. Güven, Phys. Lett. B276 (1992) 49.

[4] A. Sen, Nucl. Phys. B440 (1995) 421, hep-th/9411187;

G. T. Horowitz and A. Sen, Phys. Rev. D53 (1996) 808, hep-th/9509108.

[5] M. Cvetič and D. Youm, Phys. Rev. D53 (1996) R584, hep-th/9507090; preprint, hep-th/9512127; Nucl. Phys. B476 (1996) 118, hep-th/9603100.

[6] M. Cvetič and D. Youm, Proceedings of String '95, hep-th/9508058; Phys. Rev. D54 (1996) 2612, hep-th/9603147; Nucl. Phys. B477 (1996) 118, hep-th/9605051; preprint, hep-th/9612229.

[7] M. Cvetič and A. A. Tseytlin, Phys. Rev. D53 (1996) 5619, hep-th/ 9512031;

A. A. Tseytlin, Mod. Phys. Lett. A11 (1996) 689, hep-th/9601177.

[8] A. Strominger and C. Vafa, Phys. Lett. B379 (1996) 99, hep-th/9601029; A. Strominger, Phys. Lett. B383 (1996) 39, hep-th/9602111.

[9] C. G. Callan and J. M. Mardacena, Nucl. Phys. B472 (1996) 591, hep-th/9602043.

[10] J. C. Breckenridge, R. C. Myers, A. W. Peet and C. Vafa, preprint, hep-th/9602065; J. C. Breckenridge, D. A. Lowe, R. C. Myers, A. W. Peet, A. Strominger and C. Vafa, Phys. Lett. B381 (1996) 423, hep-th/9603078.

[11] J. Maldacena and L. Susskind, Nucl. Phys. B475 (1996) 679, hep-th/9604042.

[12] J. M. Maldacena and A. Strominger, Phys. Rev. Lett. 77 (1996), hep-th/9603060.

[13] C. V. Johnson, R. R. Khuri and R. C. Myers, Phys. Lett. B378 (1996) 78, hepth/9603061. 
[14] G. Horowitz and A. Strominger, Phys. Rev. Lett. 77 (1996) 2368, hep-th/9602051.

[15] G. T. Horowitz, J. M. Maldacena and A. Strominger, Phys. Lett. B383 (1996) 151, hep-th/9603109.

[16] G. T. Horowitz, D. A. Lowe and J. M. Maldacena, Phys. Rev. Lett. 77 (1996) 430, hep-th/9603195.

[17] G. Papadopoulos and P. Townsend, Phys. Lett. B380 (1996) 273, hep-th/9603087.

[18] A. A. Tseytlin, Nucl. Phys. B475 (1996)149, hep-th/9604035.

[19] M. J. Duff, H. Lü and C. N. Pope, Phys. Lett. B382 (1996) 73, hep-th/9604052.

[20] H. Lü, C. N. Pope, E. Sezgin and K. S. Stelle, Nucl. Phys. B456 (1996) 669, hepth/9508042; N. Khviengia, Z. Khviengia, H. Lü and C. N. Pope, Phys. Lett. B388 (1996) 21, hep-th/9605077.

[21] I. R. Klebanov and A. A. Tseytlin, Nucl. Phys. B475 (1996) 164, hep-th/9604089.

[22] I. R. Klebanov and A. A. Tseytlin, Nucl. Phys. B475 (1996) 179, hep-th/9604166.

[23] M. Cvetič and A. A. Tseytlin, Nucl. Phys. B478 (1996) 181, hep-th/9606033.

[24] J. P. Gauntlett, D. A. Kastor and J. Traschen, Nucl. Phys. B478 (1996) 544, hepth/9604179.

[25] M. J. Duff and J. Rahmfeld, Nucl. Phys. B481 (1996) 332, hep-th/9605085.

[26] M. Cvetič and C. M. Hull, Nucl. Phys. B480 (1996) 296, hep-th/9606193.

[27] D. Garfinkle, Phys. Rev. D46 (1992) 4286.

[28] R. C. Myers and M. J. Perry, Ann. Phys. 172 (1986) 304.

[29] V. Balasubramanian and F. Larsen, Nucl. Phys. B478 (1996) 199, hep-th/9604189.

[30] K. Behrndt, E. Bergshoeff, Phys. Lett. B383 (1996) 383, hep-th/9605216.

[31] M. S. Costa, preprints, hep-th/9609181, hep-th/9610138. 